\title{
A Functional Asymmetry in the Leech Heartbeat Timing Network Is Revealed by Driving the Network across Various Cycle Periods
}

\author{
Mark A. Masino and Ronald L. Calabrese \\ Biology Department, Emory University, Atlanta, Georgia 30322
}

We tested predictions of a computational model (Hill et al., 2002) of the leech heartbeat timing network. The timing network consists of two segmental oscillators located in the third (G3) and fourth (G4) segmental ganglia. Each oscillator consists of two reciprocally inhibitory oscillator interneurons along with the coordinating interneuron fibers that link them. In the model, the network was driven to cycle periods around the normal period of the network by repeatedly stimulating one of the paired oscillator interneurons in G3 or G4. Here we replicate these experiments in the biological system.

The model predicts that the G3 and G4 oscillators can entrain the timing network to periods faster but not slower than the inherent period of the nondriven ("follower") oscillator and that they can do so symmetrically. The biological system can be driven to periods both faster (such that the driven oscillator leads in phase) and slower (such that the driven oscillator lags in phase) than the inherent period of the timing network. Although both oscillators can entrain the network, the G4 oscillator does so over a narrower range of periods. Two differences between the assumptions of the model and the properties of the biological network, spike frequency adaptation in coordinating interneurons and asymmetry in the connections from the oscillator interneurons to the coordinating interneurons, may account for these discrepancies.

Individual coordinating interneurons were also able to entrain the oscillators but with little effect of the phase relationship between the oscillators, suggesting that phase relations are determined by properties inherent to the oscillator interneurons.

Key words: neuronal oscillator; central pattern generator; Hirudo medicinalis; neural network; entrainment; phase
Coupled neuronal oscillators form the basis of many motor pattern-generating networks in invertebrates and vertebrates (Marder and Calabrese, 1996). Several experimental and modeling studies have aimed at elucidating the mechanisms by which the phase relationships among these oscillators are established (Stein, 1971; Cohen, 1987; Wallén et al., 1992; Grillner et al., 1993; Sigvardt, 1993; Braun and Mulloney, 1995; Mulloney, 1997; Wadden et al., 1997; Skinner and Mulloney, 1998; Kotaleski et al., 1999a,b). These studies often focus on determining whether the phase differences arise from differences in the intrinsic excitability or period of the oscillators, or from asymmetries in network connectivity.

The timing network of the leech heartbeat central pattern generator consists of two coupled segmental oscillators in the third (G3) and fourth (G4) segmental ganglia of the ventral nerve cord (Calabrese et al., 1995). When this timing network is isolated from the rest of the nerve cord, the G3 and G4 oscillators display flexible phase relationships (Masino and Calabrese, 2002a) in contrast to the constant phase relationships observed in other systems of coupled segmental oscillators where phase differences appear to be caused by network asymmetries (Cohen, 1987; Sigvardt, 1993; Wadden et al., 1997; Skinner and Mulloney, 1998). We have previously shown that inherent period differences between the heartbeat segmental oscillators occur, the faster

Received Nov. 9, 2001; revised March 20, 2002; accepted March 22, 2002.

This work was supported by National Institutes of Health Grant NS24072. We thank Dr. Andrew A. V. Hill for the bespoke Matlab scripts provided for data analysis. Also, we thank Dr. Angela Wenning and Anne-Elise Tobin for their critical evaluations of this manuscript.

Correspondence should be addressed to Ronald L. Calabrese, Biology Department, Emory University, 1510 Clifton Road, Atlanta, GA 30322. E-mail: rcalabre@biology.emory.edu.

Copyright (C) 2002 Society for Neuroscience $\quad 0270-6474 / 02 / 224418-10 \$ 15.00 / 0$ segmental oscillator leads in phase, the magnitude of the phase difference is proportional to the period difference between the segmental oscillators, and the period of the coupled system is that of the faster segmental oscillator (Masino and Calabrese, 2002a,b). Nevertheless, there are asymmetries in the inhibitory connections from the G3 and G4 oscillator interneurons that produce the oscillations to the coordinating interneurons that link the segmental oscillators (Peterson, 1983a,b; Masino and Calabrese, 2002a). A conductance-based model of the timing network that ignores the asymmetries in network connectivity (simple symmetric model) is consistent with the experimental findings thus far (Hill et al., 2002). This model suggests that the leading oscillator speeds the following oscillator to its period by relieving inhibition from the coordinating interneurons. Our previous experimental studies were performed under conditions where the segmental oscillators were either experimentally uncoupled or free to interact normally (closed-loop conditions) and were thus mutually entrained.

Here we test the network under open-loop conditions. One oscillator interneuron from G3 or G4 was driven with rhythmic current pulses to a new period different from the mutually entrained system so that the system became entrained to the driven period. Because the current pulses controlled the driven oscillator, it was essentially insensitive to feedback from the follower oscillator. Under these open-loop conditions, asymmetries between the segmental oscillators were revealed that correspond to the asymmetries in network connectivity. Moreover, it was possible to entrain the timing network to driven periods where the driven oscillator lagged the follower oscillator. The driven oscillator lagging the follower oscillator cannot be accounted for by the simple mechanism of removal of coordinating interneuron inhibition from the slower oscillator by the faster oscillator (Hill 
A

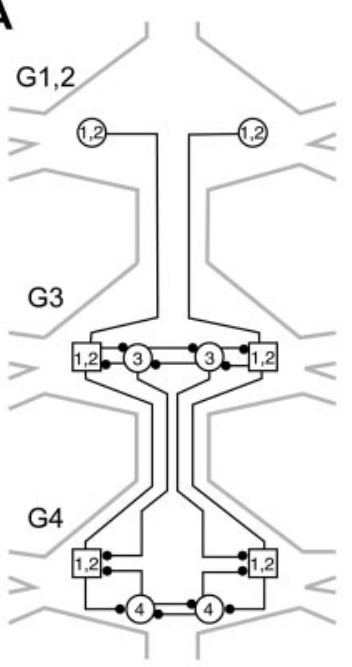

B Simple Symmetrical Model

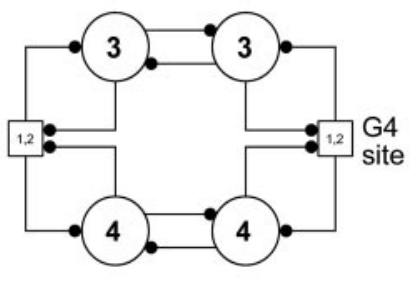

C Simple Asymmetrical Model

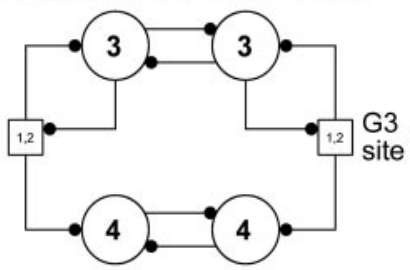

D

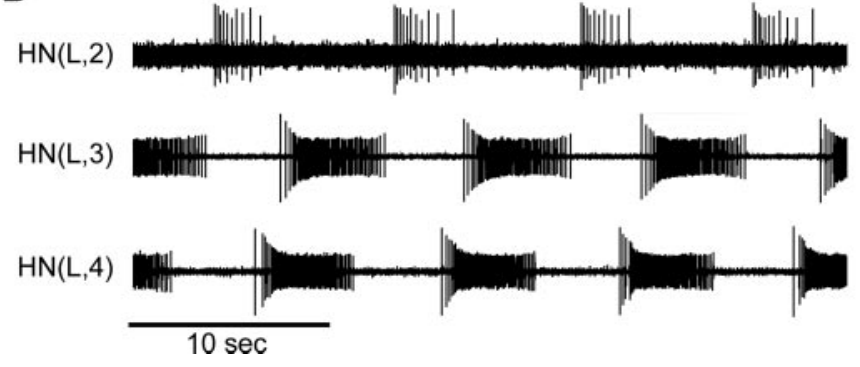

Figure 1. Circuit diagram and electrical activity of the leech heartbeat timing network. $A$, The timing network consists of paired heart (HN) interneurons in the first four segmental ganglia (G1-G4). The first and second ganglia are represented as a single ganglion for simplicity. Open circles denote somata, solid lines are cell processes, squares are distal sites of spike initiation, and filled circles are inhibitory synapses. Numbers identify the segmental ganglion where the heart interneuron somata are located. $B$, Simple symmetrical model of the timing network. All neurons are represented by a single isopotential compartment. Open circles represent oscillator interneuron somata, squares are coordinating interneuron spike initiation sites, and filled circles are inhibitory synapses. The coordinating interneurons are modeled with a single site of spike initiation represented here as the G4 site. In the symmetrical model, both the G3 and G4 oscillator interneurons inhibit the coordinating interneuron spike initiation site. $C$, Simple asymmetrical model of the timing network. Somata, spike initiation sites, and synapses are represented as in $B$. The coordinating interneurons are modeled with a single site of spike initiation represented here as the G3 site. In the asymmetrical model, only the G3 oscillator interneurons inhibit the coordinating interneuron spike initiation site. $D$, Coordinated activity of ipsilateral heart interneurons in G2 through G4. The oscillator interneurons in G3 and G4 are active nearly in-phase, whereas the coordinating interneuron is active in anti-phase.

et al., 2002). Analysis of network activity when driving coordinating interneurons and when oscillator interneurons entrainment broke down led to further insights into network function.

\section{MATERIALS AND METHODS}

Animals and solutions. Leeches (Hirudo medicinalis) were obtained from commercial suppliers (Leeches USA, Westbury, NY and Biopharm, Charleston, NC) and maintained in artificial pond water at $15^{\circ} \mathrm{C}$. After the animals were anesthetized in cold saline, ganglia were dissected and pinned (ventral surface up) in small Petri dishes filled with Sylgard (Dow Corning, Midland, MI). Ganglia were desheathed using fine scissors. Heart interneurons were identified based on soma size, soma location in the ganglion, and ultimately by their characteristic bursting activity (Fig. $1 D)$. The desheathed preparation was superfused continuously with normal leech saline containing (in mM): $115 \mathrm{NaCl}, 4 \mathrm{KCl}, 1.8 \mathrm{CaCl}_{2}, 10$ glucose, and 10 HEPES buffer, adjusted to $\mathrm{pH} 7.4$ with $\mathrm{NaOH}$. Depending on the experimental protocol used, preparations consisted of chains of ganglia either from the head brain to fourth ganglion (HB-G4) or from the third to fourth ganglia (G3-G4).

Extracellular recording techniques. For extracellular recordings, we used suction electrodes to record the heart interneurons following the methods described in Masino and Calabrese (2002a).

Intracellular recordings and stimulating techniques. For intracellular recording and stimulation, we used sharp intracellular electrodes ( $\sim 20-25 \mathrm{M} \Omega$ filled with $4 \mathrm{~m} \mathrm{~K}$ acetate, $20 \mathrm{~mm} \mathrm{KCl}$ ) following the methods described in Nadim and Calabrese (1997). For intracellular stimulation ("driving") of heart interneurons to periods faster and slower than the normal cycle period of the timing network, we passed depolarizing current pulses (between 0.1 and $1 \mathrm{nA} ; 50 \%$ duty cycle) over a range of periods into the penetrated cell. Square wave pulses were generated with a Wavetek (model 75; San Diego, CA) arbitrary waveform generator, which gated a user determined current (step command) from an Axoclamp 2A amplifier (Axon Instruments, Foster City, CA). The depolarizing current pulses were superimposed on a constant holding current of negative polarity (typically between -0.1 and $-0.5 \mathrm{nA}$ ) that ensured that the cell did not spike during the inactive portion (trough of the square wave stimulation) of the burst cycle. The current amplitude was adjusted continuously to set and maintain a current sufficient to produce high-frequency firing of the cell during the upswing of the square wave. The change between the driven and the normal cycle periods were defined as:

$$
\left(\left(\frac{T_{\text {Driven }}-T_{\text {Normal }}}{T_{\text {Normal }}}\right) \times 100\right)
$$

Control data for cycle period, phase, and duty cycle were collected for each preparation before experimental manipulation (stimulation).

Data acquisition and analysis. Data (extracellular and intracellular recordings) were digitized using a digitizing board (DigiData 1200 Series Interface; Axon Instruments) and acquired using pClamp software (Axon Instruments) on a personal computer. A spike train analysis program, written in Matlab (Mathworks, Natick, MA), was used to analyze the data on a personal computer.

Spikes were detected following the methods described in Masino and Calabrese (2002a). Once spikes were detected, they were grouped into bursts as follows. After an interburst interval $(1 \mathrm{sec})$ elapsed without any spikes detected, the next spike event was identified as the first spike of a burst. Subsequent spikes with interspike intervals less than the interburst interval $(<1 \mathrm{sec})$ were grouped into that burst. To eliminate the effects of stray spikes in oscillator interneurons, groups of less than five spikes were not considered as bursts. In coordinating interneurons, which had fewer spikes per burst than oscillator interneurons, groups of at least two spikes were considered bursts. In the electrophysiological recordings, the median spike in each burst was indicated by a symbol above the burst. Symbols represent heart interneurons from specific ganglia: diamond, G2 coordinating interneurons; circle, G3 oscillator interneurons; asterisk, G4 oscillator interneurons; and square, contralateral oscillator interneuron in the same ganglion as the driven cell (either G3 or G4). In some of the driving experiments where breakdowns in one-to-one entrainment occurred, bursts had to be recognized subjectively because subsequent bursts sometimes ran into one another.

The analysis program was also used to determine cycle period $(T)$, phase $(\phi)$, and duty cycle $(D)$ for each recorded cell $(n \geq 12$ consecutive bursts per cell). Cycle period was defined as the interval in seconds from median spike to median spike of consecutive bursts, and the mean cycle period $\left(T_{X}\right)$ was determined for each cell $(X)$. The phase of a given heart interneuron was defined 
on a cycle-by-cycle basis as the time $(t)$ difference between its median spike $\left(t_{X}\right)$ and the median spike of a G4 oscillator interneuron $\left(t_{4}\right.$; phase marker cell). The time difference $(\Delta t)$ was then normalized to the cycle period of the phase marker cell and expressed as a percentage:

$$
\left(\phi_{\mathrm{X}}=\left(\frac{\Delta t_{\mathrm{x}-4}}{T_{4}}\right) \times 100\right) .
$$

A phase of 100/0\% indicated a cell with no phase difference relative to the phase marker cell, whereas a $50 \%$ phase difference indicated an anti-phasic relationship. A positive phase difference indicated a phase lag, whereas a negative phase difference indicated a phase lead with respect to the phase marker cell. Duty cycle $(D)$ was defined as the percentage of the cycle period occupied by the burst duration $\left(T_{\text {burstX } X}\right)$ :

$$
D=\left(\frac{T_{\text {burst } X}}{T_{X}}\right) \times 100 .
$$

Actograms illustrated the network activity and firing relationships between heart interneurons in the timing network (Fig. 2, right column). Actograms were based on raster presentations similar to those used to display circadian activity rhythms (Pittendrigh, 1974; Peterson and Calabrese, 1982). Each symbol (indexed by ganglion) represented the time of occurrence of the median spike in the burst of an interneuron. The reference cycle of the actogram was usually defined by the mean cycle period of the phase marker cell in an unmodified ganglion chain before any experimental manipulation. In cases where breakdowns in oneto-one entrainment are illustrated, the reference cycle was set to the driven period. Time was broken into a series of segments of constant length (reference cycle) that were arranged sequentially, one below the other. When the cycle period of an interneuron was equal to the segment length, then the symbols formed a straight vertical line. When the cycle period was less than the segment length, then the symbols drifted to the left; when it was greater, the symbols drifted to the right. For visual purposes, a duplicate copy of each segment was displayed to the right and shifted up one row in the graph.

Entrainment and breakdown of entrainment in the timing network. During entrainment, the bursts of the follower cells matched one-to-one with the bursts of the driven cell (indicated in physiological traces and phase actograms). One-to-one matching of symbols, which were displaced from one another by a regular horizontal interval, indicated a stable phase relationship between interneurons, i.e., one-to-one entrainment. Breakdown of entrainment between the driven and follower cells occurred when the cells cycled independently, usually at different periods, and when the activity between the cells was not phase locked (i.e., the bursts of the follower cells did not match one-to-one with the bursts of the driven cell). These driving experiments were openloop in nature because the driven oscillator interneuron effectively squelched feedback between the oscillators, but the driven oscillator was able to entrain the follower through feedforward inhibition from the coordinating interneurons.

\section{RESULTS}

The leech heartbeat central pattern generator consists of paired inhibitory heart interneurons in the first through the seventh segmental ganglia (G1-G7) (Calabrese et al., 1995). A subset of these heart interneurons, located in G1 through G4, forms the heartbeat timing network (Fig. $1 A$ ). Two foci of oscillation in the
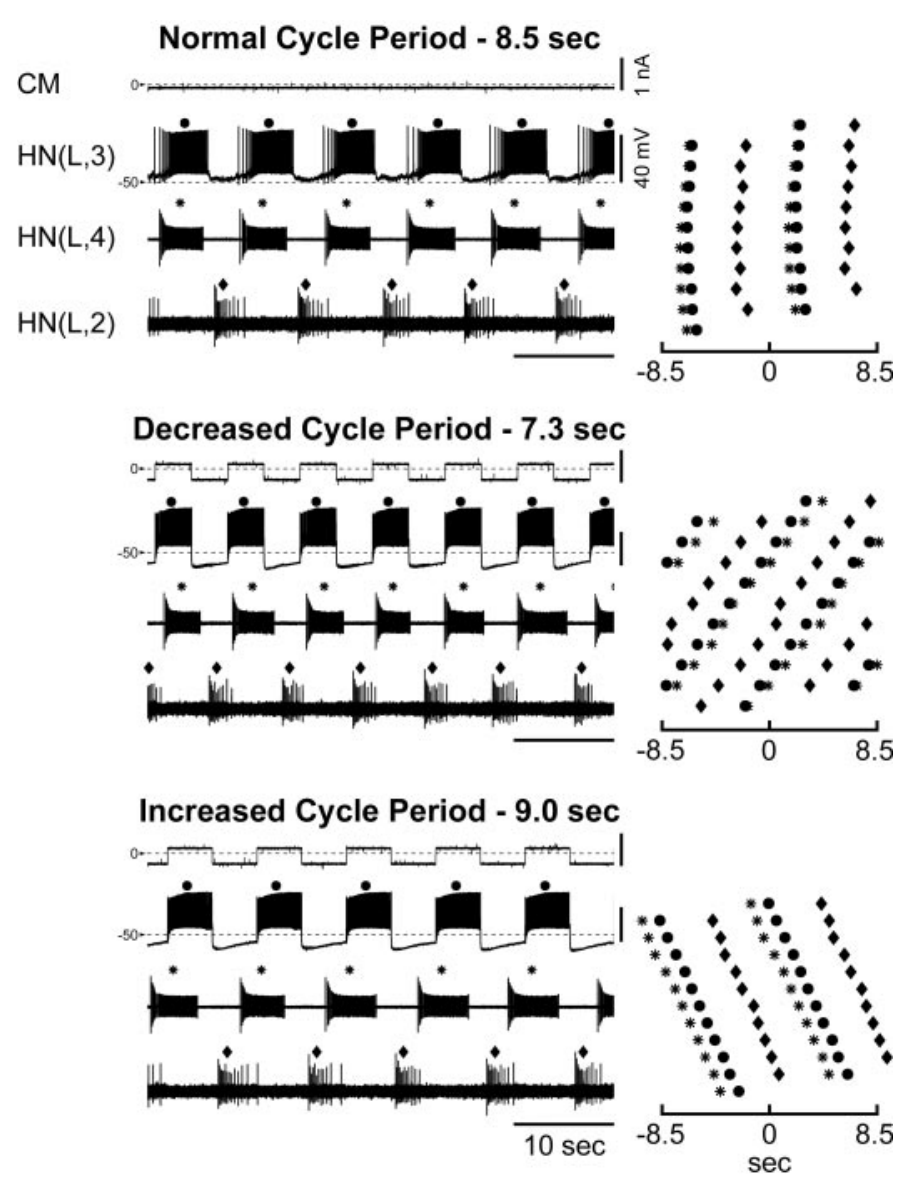

Figure 2. The timing network is entrained to periods faster and slower than the normal cycle period by driving one of the paired oscillator interneurons in G3. Simultaneous intracellular $[H N(L, 3)]$ and extracellu$\operatorname{lar}[H N(L, 4)$ and $H N(L, 2)]$ recordings of ipsilateral heart interneurons are illustrated in each panel. The median spike of each burst is indicated by a symbol, which is indexed by ganglion: circle, G3 oscillator interneuron; asterisk, G4 oscillator interneuron; diamond, G2 coordinating interneuron. Normal Cycle Period, The cycle period $(8.5 \mathrm{sec})$ of the timing network is regular, and the activity of the heart interneurons is phase locked. Regularity of the timing relationships between the heart interneurons is illustrated in the actogram to the right of the electrophysiological traces. The $\mathrm{HN}(\mathrm{L}, 4)$ interneuron leads the $\mathrm{HN}(\mathrm{L}, 3)$ interneuron in phase, whereas the coordinating $(\mathrm{HN}(\mathrm{L}, 2))$ interneuron is active in antiphase. Decreased Cycle Period, HN(L,3) interneuron is driven by current pulses to a period $(7.3 \mathrm{sec})$ that is faster than the normal cycle period $(8.5 \mathrm{sec})$. The symbols in the actogram drift to the left because the driven period is faster than the normal cycle period. The phase relationship between the G3 and G4 oscillator interneurons has reversed, such that the $\mathrm{HN}(\mathrm{L}, 3)$ interneuron now leads the $\mathrm{HN}(\mathrm{L}, 4)$ interneuron in phase. The coordinating interneuron remains in anti-phase. Increased Cycle Period, HN(L,3) is driven by current pulses to a period $(9.0 \mathrm{sec})$ that is slower than the normal cycle period $(8.5 \mathrm{sec})$. The symbols in the actogram drift to the right because the driven period of the interneuron is slower than the normal cycle period. The $\mathrm{HN}(\mathrm{L}, 4)$ interneuron phase lead over the $\mathrm{HN}(\mathrm{L}, 3)$ interneuron is larger than observed in the normal cycle period. In this and all subsequent figures, the current trace [current monitor $(C M)$ ] indicates the holding current ( 0 current indicated by arrowhead and dashed line) and the rhythmic current pulses (50\% duty cycle) applied to the intracellularly recorded heart interneuron (driven cell).

timing network have been identified in G3 and G4, where the oscillation is dominated by the reciprocal synaptic interactions of the third and fourth pair of heart interneurons, respectively (Peterson, 1983a). Reciprocally inhibitory synapses between the bilateral pairs of heart interneurons in these ganglia, combined with an ability of these interneurons to escape from inhibition, 
pace the oscillation (Peterson, 1983a; Angstadt and Calabrese, 1989; Nadim et al., 1995; Hill et al., 2001). Thus, the heart interneurons in G3 and G4 are called oscillator interneurons. The heart interneurons of $\mathrm{G} 1$ and $\mathrm{G} 2(\mathrm{G} 1,2)$ act as coordinating fibers and link the oscillator interneurons in G3 and G4, thus forming the heartbeat timing network for the system (Peterson, 1983b). The oscillator interneurons in G3 and G4 continue to oscillate normally in isolated, single ganglion preparations. Thus, each of these two reciprocally inhibitory heart interneuron pairs, along with the coordinating interneuron fibers in each ganglion, is considered an autonomous segmental oscillator (Peterson, 1983a; Hill et al., 2001).

The intersegmental phase relationships between the coupled segmental oscillators in the isolated heartbeat timing network are flexible (Masino and Calabrese, 2002a). Although the activity of the G3 to G4 oscillator interneuron observed in the isolated nerve cord preparations are phase locked within individual unperturbed preparations (Fig. 1D), they vary considerably among different preparations. These phase relationships are generated by period differences between the G3 and G4 segmental oscillators such that the inherently faster oscillator, regardless of whether it is located in G3 or G4, leads in phase and determines the cycle period of the timing network (Masino and Calabrese, 2002b).

The coordinating interneurons can initiate spikes at sites located in G4 and G3 (Peterson, 1983a; Masino and Calabrese, 2002a). Because there is an asymmetry in the synaptic connections between the G3 and G4 oscillator interneurons onto the coordinating interneurons (Fig. $1 A$ ), the timing network can potentially function in two modes, depending on where the coordinating interneurons initiate their spikes (Hill et al., 2002). The network functions in a symmetric mode if spikes originate at the initiation site in G4 because both G3 and G4 oscillator interneurons inhibit this initiation site (Fig. 1A,B). However, if spikes are initiated at the site in G3, the network functions in an asymmetric mode because only the oscillator interneurons in G3 inhibit this site (Fig. $1 A, C$ ). Although the network can potentially function in either the symmetric or asymmetric mode, the biological network appears to function mainly in the symmetric mode because the majority of the coordinating interneuron spikes $(>75 \%)$ are initiated at the spike initiation site in G4 (Masino and Calabrese, 2002a).

Our previous experiments on intersegmental coordination in the heartbeat timing network have been done where the segmental oscillators freely interact under normal conditions (closedloop) of mutual entrainment (Masino and Calabrese, 2002b; Hill et al., 2002), and the results are consistent with a conductance based model based on the symmetric mode circuit of Figure $1 C$. Here we tested the network under open-loop conditions to determine whether structural asymmetries in the network could be observed in the functional output of the network. One oscillator interneuron from G3 or G4 was driven with periodic current pulses to a new period from the mutually entrained network, and the abilities of the driven G3 and G4 oscillators to entrain the timing network across various cycle periods were compared. When the network was entrained (Figs. 2-4 ), the driven oscillator was controlled and thus was insensitive to feedback from the follower oscillator.

\section{The timing network is entrained to various cycle periods by driving a single heart interneuron}

The heartbeat timing network could be entrained to periods faster and slower than the normal cycle period when one of the
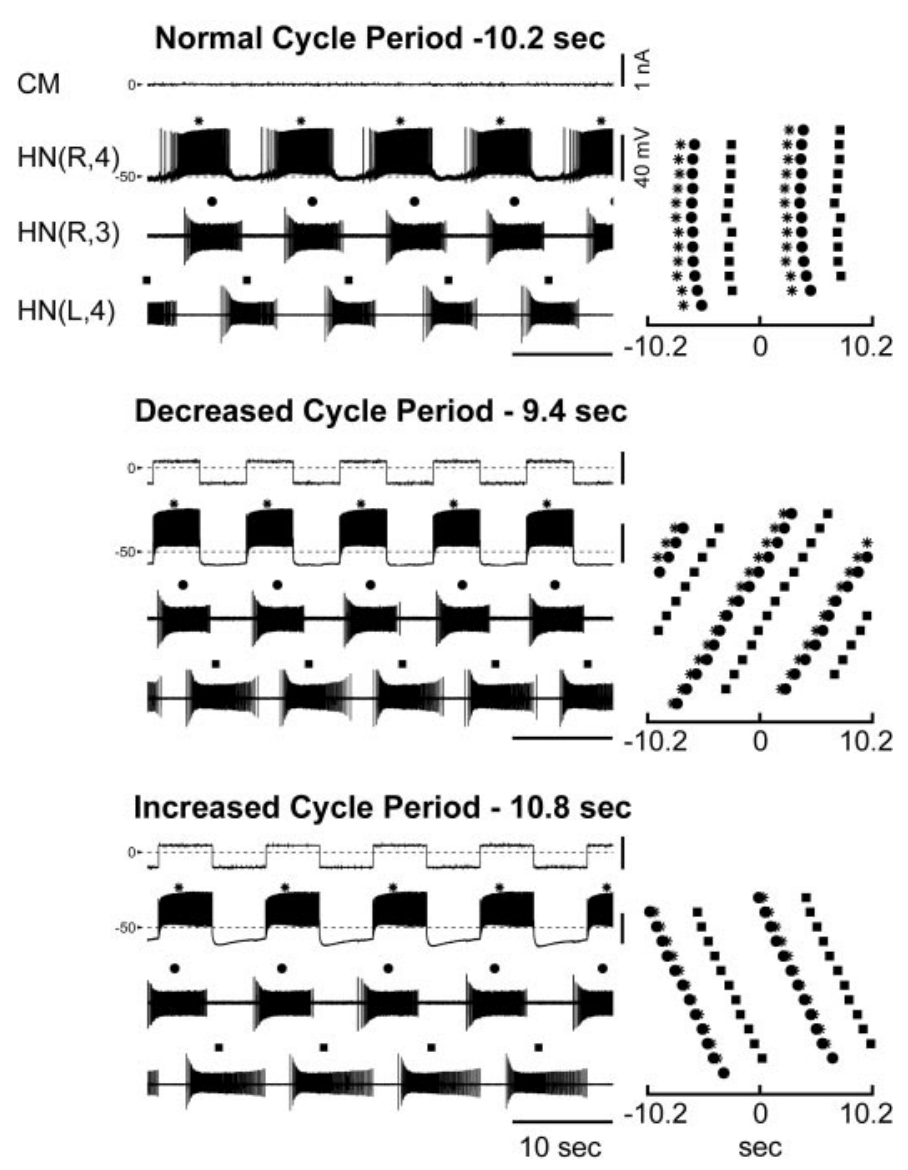

Figure 3. The timing network is entrained to periods faster and slower than the normal cycle period by driving one of the paired oscillator interneurons in G4. Simultaneous intracellular $[H N(R, 4)]$ and extracellular $[H N(R, 3)$ and $H N(L, 4)]$ recordings of oscillator interneurons are illustrated in each panel. The median spike of each burst is indicated by a symbol, which is indexed by ganglion: asterisk, G4 oscillator interneuron; circle, G3 oscillator interneuron; square, G4 oscillator interneuron contralateral to the driven cell. Normal Cycle Period, The cycle period $(10.2$ $\mathrm{sec}$ ) of the timing network is regular, and the activity of the heart interneurons is phase locked. Regularity of the timing relationships between the heart interneurons is illustrated in the actogram to the right of the electrophysiological traces. Decreased Cycle Period, The HN(R,4) interneuron is driven by current pulses to a period $(9.7 \mathrm{sec})$ that is faster than the normal cycle period $(10.2 \mathrm{sec})$. The symbols in the actogram drift to the left because the driven period is faster than the normal cycle period. Increased Cycle Period, The $\mathrm{HN}(\mathrm{R}, 4)$ interneuron is driven by current pulses to a period $(10.8 \mathrm{sec})$ that is slower than the normal cycle period $(10.2 \mathrm{sec})$. The symbols in the actogram drift to the right because the driven period is slower than the normal cycle period.

paired oscillator interneurons in G3 was driven with periodic current pulses (Fig. 2). Before stimulation ("undriven"), the recorded cells of the timing network cycled at the same period and were phase locked (Fig. 2, Normal Cycle Period). The regularity of the cycle period and the phase relationships are shown in the phase actogram to the right in Figure 2. Note that all cells cycled at the same period and that the G4 oscillator interneuron $[(\mathrm{HN}(\mathrm{L}, 4)]$ led the $\mathrm{G} 3$ oscillator interneuron $[\mathrm{HN}(\mathrm{L}, 3)]$ in phase; the $\mathrm{G} 2$ coordinating interneuron $[\mathrm{HN}(\mathrm{L}, 2)]$ was in anti-phase. Current pulses with a period less than the normal cycle period delivered to the driven cell $[\mathrm{HN}(\mathrm{L}, 3)]$ sped up the timing network (Fig. 2, Decreased Cycle Period). The network assumed the period of the driven (faster) cell and the normal (undriven) G3 to G4 phase relationship reversed, such that the driven (faster) cell led 


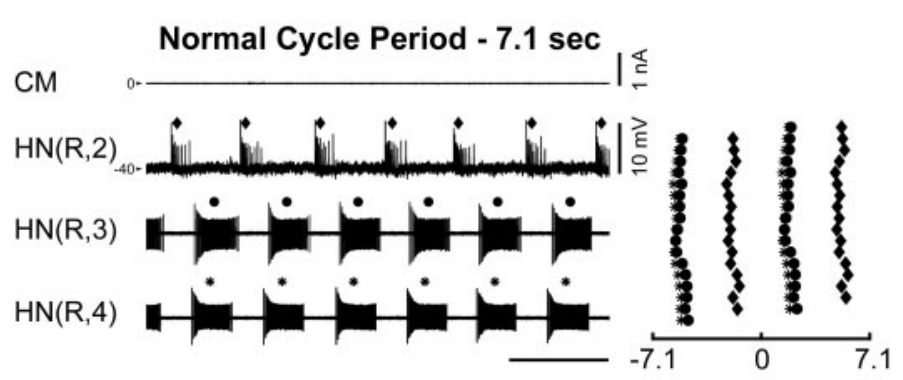

Decreased Cycle Period - $6.8 \mathrm{sec}$
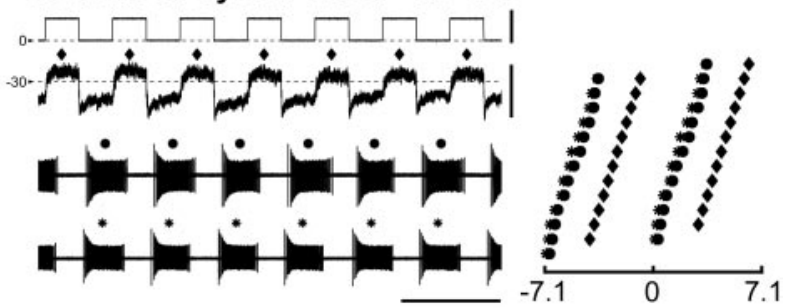

Increased Cycle Period - 7.4 sec
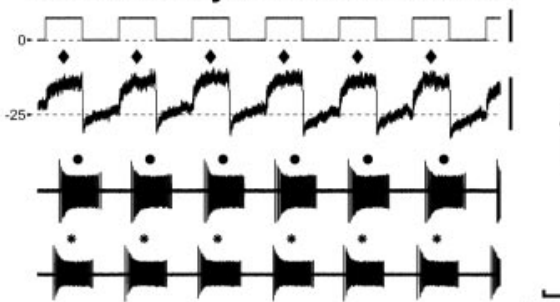

$10 \mathrm{sec}$

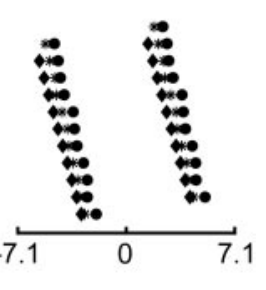

Figure 4. The timing network is entrained to periods faster and slower than the normal cycle period by driving one of the paired coordinating interneurons in G2. Simultaneous intracellular $[H N(R, 2)]$ and extracellular $[H N(R, 3)$ and $H N(R, 4)]$ recordings of ipsilateral heart interneurons are illustrated in each panel. The median spike of each burst is indicated by a symbol, which is indexed by ganglion: diamond, G2 coordinating interneuron; circle, G3 oscillator interneuron; asterisk, G4 oscillator interneuron. Normal Cycle Period, The cycle period $(7.1 \mathrm{sec})$ of the timing network is regular, and the activity of the heart interneurons is phase locked. Regularity of the timing relationships between the heart interneurons is illustrated in the actogram to the right of the electrophysiological traces. The $\operatorname{HN}(R, 4)$ interneuron leads the $H N(R, 3)$ interneuron in phase, whereas the coordinating interneuron $[\mathrm{HN}(\mathrm{R}, 2)]$ is active in antiphase. Decreased Cycle Period, The $\mathrm{HN}(\mathrm{R}, 2)$ interneuron is driven by current pulses to a period $(6.8 \mathrm{sec})$ that is faster than the normal cycle period $(7.1 \mathrm{sec})$. Notice that the spikes generated by injecting current pulses into the coordinating interneuron soma are very small and usually lost in the noise as first noted by Peterson (1983b). The symbols in the actogram drift to the left because the driven period is faster than the normal cycle period. The phase relationship between the G3 and G4 oscillator interneurons does not change, and the coordinating interneuron remains in approximate anti-phase. Increased Cycle Period, The $\mathrm{HN}(\mathrm{R}, 2)$ interneuron is driven by current pulses to a period $(7.4 \mathrm{sec})$ that is slower than the normal cycle period $(7.1 \mathrm{sec})$. The symbols in the actogram drift to the right because the driven period is slower than the normal cycle period. There is a slight increase in the phase lead of the $\operatorname{HN}(\mathrm{R}, 4)$ interneuron over the $\mathrm{HN}(\mathrm{R}, 3)$ interneuron. Notice that the timing of the current pulse in the coordinating interneuron $[H N(R, 2)]$ is nearly in phase with the activity of the oscillator interneurons $[H N(R, 3)$ and $H N(R, 4)]$.

in phase. The coordinating interneuron $[\mathrm{HN}(\mathrm{L}, 2)]$ remained in anti-phase. The timing network was slowed by applying current pulses with a period greater than the normal cycle period to the driven cell [HN(L,3)] (Fig. 2, Increased Cycle Period). At this increased period, the network assumed the period of the driven (slower) cell. The nondriven (follower) cell in $\mathrm{G} 4$ [HN(L,4)], which was presumably faster, led in phase. The G3 to G4 phase relationship at the increased cycle period was similar to, but greater than the phase relationship at the normal cycle period. At both the normal and slow periods, the nondriven cell in G4 $[\mathrm{HN}(\mathrm{L}, 4)]$ presumably had the faster cycle period. Equivalent results were obtained when recording cells opposite to, but in the same ganglion as the driven cell, which indicated that the entire network was entrained.

The heartbeat timing network also could be entrained to a range of periods faster and slower than the normal cycle period when one of the paired oscillator heart interneurons in G4 was driven with periodic current pulses (Fig. 3). Before stimulation, the recorded cells of the timing network cycled at the same period and were phase locked (Fig. 3, Normal Cycle Period). The regularity of the cycle period and the phase relationships are shown in the phase actogram to the right in Figure 3. Note that all cells cycled at the same period and that the G4 oscillator interneuron $[\mathrm{HN}(\mathrm{R}, 4)]$ led the $\mathrm{G} 3$ oscillator interneuron $[\mathrm{HN}(\mathrm{R}, 3)]$ in phase; the contralateral $\mathrm{G} 4$ oscillator interneuron $[\mathrm{HN}(\mathrm{L}, 4)]$ was in anti-phase to $[\mathrm{HN}(\mathrm{R}, 4)]$. Current pulses with a period less than the normal cycle period applied to the driven cell $[\mathrm{HN}(\mathrm{R}, 4)]$ sped up the timing network (Fig. 3, Decreased Cycle Period). The network assumed the period of the driven (faster) cell. In addition, the driven (faster) cell led in phase, whereas the contralateral oscillator interneuron $[\mathrm{HN}(\mathrm{L}, 4)]$ remained in anti-phase. The timing network was slowed by applying current pulses with a period greater than the normal cycle period to the driven cell $[\mathrm{HN}(\mathrm{R}, 4)]$ (Fig. 3, Increased Cycle Period). At this increased period, the network assumed the period of the driven (slower) cell. The original G3 to G4 phase relationship was reversed, such that the follower cell $[\mathrm{HN}(\mathrm{R}, 3)]$ led in phase, presumably because it had a faster cycle period. Equivalent results were obtained when recording coordinating interneurons ipsilateral to the driven cell, which indicated that the entire network was entrained.

Finally, the heartbeat timing network could be entrained to a range of periods faster and slower than the normal cycle period when one of the paired coordinating heart interneurons in G2 was driven with periodic current pulses (Fig. 4). Before stimulation, the recorded cells of the timing network cycled at the same period and were phase locked (Fig. 4, Normal Cycle Period). The regularity of the cycle period and the phase relationships are shown in the phase actogram to the right in Figure 4. Note that all cells cycled at the same period and that the G4 oscillator interneuron $[\mathrm{HN}(\mathrm{R}, 4)]$ slightly led the $\mathrm{G} 3$ oscillator interneuron $[\mathrm{HN}(\mathrm{R}, 3)]$ in phase; the $\mathrm{G} 2$ coordinating interneuron $[\mathrm{HN}(\mathrm{R}, 2)]$ was in antiphase to the oscillator interneurons. Current pulses applied to the driven cell $[\mathrm{HN}(\mathrm{R}, 2)]$ with a period less than the normal cycle period sped up the timing network (Fig. 4, Decreased Cycle Period). The network assumed the period of the driven (faster) cell. The G3 to G4 phase relationship at this faster period was not altered from the original phase relationship at the normal (undriven) cycle period, whereas the driven G2 coordinating interneuron $[\mathrm{HN}(\mathrm{R}, 2)]$ remained in anti-phase. The period of the timing network was slowed by applying current pulses with a period greater than the normal cycle period to the driven cell [HN(R,2)] (Fig. 4, Increased Cycle Period). At this increased period, the network assumed the period of the driven (slower) cell. Again, the G3 to G4 phase relationship $[\mathrm{HN}(\mathrm{R}, 4)$ led $\mathrm{HN}(\mathrm{R}, 3)]$ was nearly the same as the original phase relationship, but slightly larger. 


\section{Comparison of the range of entrainment}

To indicate the range of entrainment and determine the effects of driving the timing network to a new cycle period on the G3 to G4 phase relationship, we plotted the observed G3 to G4 phase relationships $\left(\phi_{3}-\phi_{4}\right)$ against the change in period

$$
\left(\Delta_{T}=\left(\frac{T_{\text {Driven }}-T_{\text {Normal }}}{T_{\text {Normal }}}\right) \times 100\right)
$$

of the timing network, which was expressed as a percentage of the normal cycle period, at each driven period (Fig. 5A-C). Taken together, the combined plots for the individual preparations in each panel indicate the range of entrainment for the driven cell type in that panel. Although the limits of entrainment at both ends (faster and slower than the normal cycle period) of the entrainment range were reached in some preparations, the limit of entrainment at just one end of the range was reached in others, because the intracellular recording of the driven cell was lost through mechanical disturbance or injury. These plots were made from data collected from HB-G4 chains because the timing network was intact in these preparations.

In six preparations, we drove a G3 oscillator interneuron with current pulses to various cycle periods and measured the resulting G3 to G4 phase relationships (Fig. $5 A$ ). The timing network remained intact when a $\mathrm{G} 3$ cell was driven across a wide range of cycle periods faster (approximately $-15 \%$ ) and slower (approximately $+15 \%$ ) than the undriven normal cycle period (Fig. 5A). The G3 to G4 phase relationships among these preparations ranged between approximately $-20 \%$ (G3 leads) to $+20 \%$ (G4 leads) over this range of driven periods. The G3 oscillator led the G4 oscillator when it was driven faster than the normal cycle period of the undriven preparation; the faster the G3 oscillator was driven, the more it led. Conversely, the G3 oscillator lagged the G4 oscillator when it was driven slower than the normal cycle period of the undriven preparation; the slower the G3 oscillator was driven, the more it lagged. In preparations in which G4 normally led in phase, driving a G3 oscillator interneuron to periods faster than the normal cycle period decreased the G4 phase lead and could reverse the phase relationship (G3 lead), whereas driving it to slower periods increased the G4 phase lead. In preparations in which where G3 normally led in phase, driving a G3 oscillator to periods faster than the normal cycle period increased the G3 phase lead, whereas driving it to slower periods decreased the G3 phase lead and could reverse the phase relationship (G4 lead).

In nine preparations, we drove a G4 oscillator interneuron with current pulses to various cycle periods and measured the resulting G3 to G4 phase relationships (Fig. $5 B$ ). The timing network remained intact when a G4 cell was driven across a narrower range (compared with the driven G3 preparations) of cycle periods faster (approximately $-5 \%$ ) and slower (approximately $+10 \%$ ) than the undriven normal cycle period (Fig. $5 B$ ). Generally, the G3 to G4 phase relationships among these preparations ranged between approximately $-20 \%$ (G3 leads) to $+20 \%$ (G4 leads) over this range of driven periods. The $\mathrm{G} 4$ oscillator led the G3 oscillator when it was driven faster than the normal cycle period of the undriven preparation; the faster the G4 oscillator was driven, the more it led. Conversely, the G4 oscillator lagged the G3 oscillator when it was driven slower than the normal cycle period of the undriven preparation; the slower the G4 oscillator was driven, the more it lagged. In preparations in which G4 normally led in phase, driving a G4 oscillator to periods faster than the normal cycle period increased the G4 phase lead, whereas driving it to slower periods decreased the G4 phase lead and could reverse the phase relationship (G3 lead). In preparations in which G3 normally led in phase, driving a G4 oscillator to periods faster than the normal cycle period decreased the G3 phase lead and could reverse the phase relationship (G4 lead), whereas driving it to slower periods increased the G3 phase lead.

The driven G3 and driven G4 preparations produced a similar range of phase relationships $(-20$ to $+20 \%)$ across different ranges of driven cycle periods $(-15$ to $+15 \%$ and -5 to $+10 \%$, respectively) (Fig. 5, compare $A, B$ ). When preparations were driven to periods slower than the normal cycle period, the mean greatest change in period at which one-to-one entrainment was observed was not significantly different $(t=-0.28 ; p=0.79)$ between driven G3 $(5.6 \pm 6.1 \%)$ and driven G4 $(6.3 \pm 4.1 \%)$ oscillators (Fig. $5 A, B$, right half of graphs). However, when preparations were driven to periods faster than the normal cycle period, a significant difference $(t=-3.1 ; p<0.01)$ in the abilities of the driven G3 $(-8.4 \pm 4.9 \%)$ and driven G4 $(-2.8 \pm 2.2 \%)$ oscillators was observed (Fig. $5 A, B$, left half of graph). Because in these driving experiments the relation between the G3 to G4 phase difference with the period difference was apparently changed, we performed linear regression for the individual experiments. The mean values of the slopes of the individual linear regression lines for driven G3 and driven G4 oscillators for the data in Figure 5, $A$ and $B$, were significantly different $(t=-9.7$; $p<0.001)$. The phase difference between the two oscillators was more sensitive to a change in driving period when a G4 oscillator was driven, compared with a G3 oscillator.

Overlap in firing between the driven cell and its contralateral partner (nondriven cell) was often observed at the limits of the entrainment range, regardless of which oscillator (G3 or G4) was driven. This overlap, however, was most evident in preparations in which the G4 oscillator was driven to slow periods (Fig. 3, Increased Cycle Period). Overlap occurred because the nondriven G4 oscillator interneuron escapes from inhibition presumably through a hyperpolarization activated inward current $\left(I_{\mathrm{h}}\right)$ (Angstadt and Calabrese, 1989). The overlap in firing between the driven G4 oscillator interneuron and its nondriven contralateral partner increases as the period of the driven cell increases because firing in the nondriven cell initiates earlier in the burst of the driven cell. This increase in overlap is reflected in a reduction of the phase difference between the oscillator interneurons of the driven half-center to $<50 \%$.

In six preparations, we drove a G2 oscillator interneuron with current pulses to various cycle periods and measured the resulting G3 to G4 phase relationships (Fig. 5C). The timing network remained intact when a $\mathrm{G} 2$ cell was driven across a moderate range of cycle periods faster (approximately -15\%) and slower (approximately $+5 \%$ ) than the undriven normal cycle period (Fig. 5C). Generally, the G3 to G4 phase relationships among these preparations remained relatively constant as the driven period changed. In preparations in which G4 normally led in phase, driving a G2 coordinating interneuron to periods faster than the normal cycle period slightly decreased the G4 phase lead, whereas driving it to slower periods slightly increased the G4 phase lead. In preparations in which G3 normally led in phase, driving a $\mathrm{G} 2$ coordinating interneuron to periods faster than the normal cycle period slightly increased the G3 phase lead, whereas driving it to slower periods slightly decreased the G3 phase lead. Overall, there was little influence, however, on the G3 to G4 phase relationship of driving a $\mathrm{G} 2$ coordinating interneuron. 


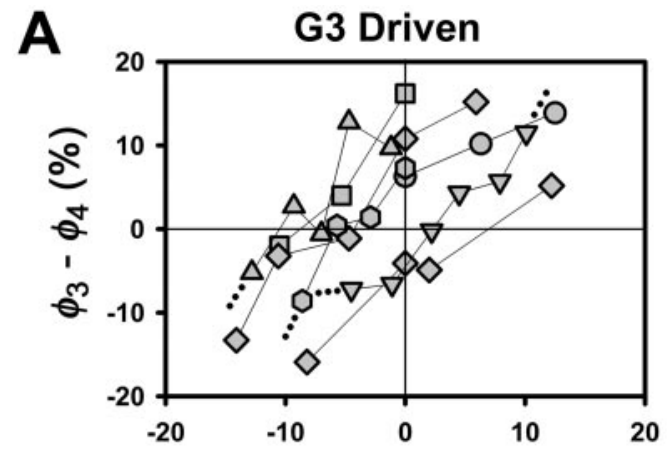

B

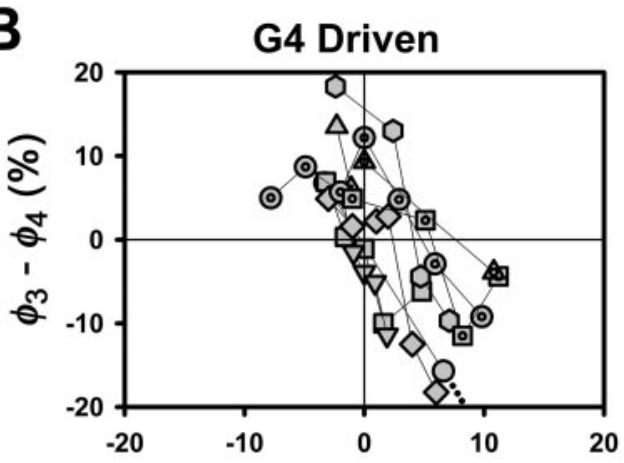

C

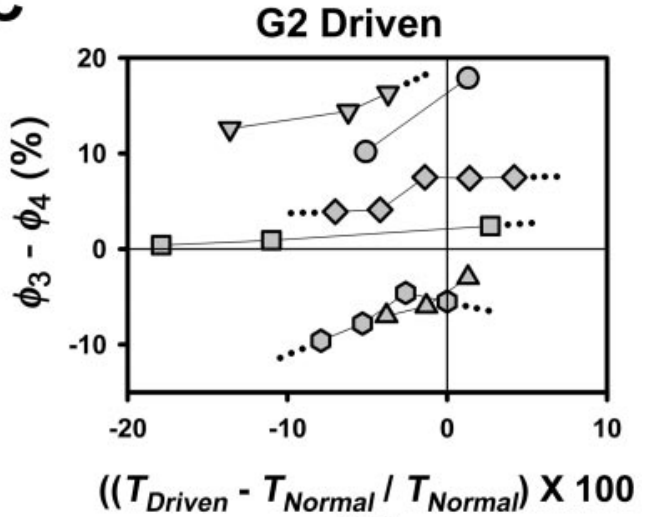

(\%)

Figure 5. Plots of the G3 to G4 phase relationships versus changes in the timing network cycle period. In each preparation, one of the paired oscillator interneurons in $\mathrm{G} 3(A)$ or $\mathrm{G} 4(B)$ or in a $\mathrm{G} 2$ coordinating interneuron $(C)$ is driven to periods both faster and slower than the normal cycle period. The G3 to G4 phase relationship $\left(\phi_{3}-\phi_{4}\right)$ at each driven period is plotted against the change in period

$$
\left(\Delta_{T}=\left(\frac{T_{\text {Driven }}-T_{\text {Normal }}}{T_{\text {Normal }}}\right) \times 100\right)
$$

of the timing network, which is expressed as a percentage of the normal cycle period. A positive phase relationship indicates that the G4 oscillator leads in phase, whereas a negative phase relationship indicates that the G3 oscillator leads in phase. The sign of the change in period indicates whether the timing network is driven to periods faster $(-)$ or slower $(+)$ than the normal cycle period. Lines that end in a symbol indicate that the limit of entrainment was reached at that end of the entrainment range, whereas lines that end in a symbol followed by a series of three dots extending from the symbol indicate that the limit of entrainment was not reached at that end of the entrainment range. The points are connected by linear segments. $A, \mathrm{G} 3$ oscillator interneurons driven to various cycle periods entrain the timing network over a wide range of periods faster (approximately $-15 \%$ ) and slower (approximately $+15 \%$ ) than the normal cycle period. There is a near linear relationship (moderate slope) between the G3 to $\mathrm{G} 4$ phase relationships and the changes in cycle period.
Breakdown in the timing network occurs at periods both faster and slower than the normal cycle period

One-to-one entrainment broke down ("breakdown") when the driven cell, and thus the network, was driven to periods either faster or slower than the range of entrainment seen in Figure $5 A-C$. Detailed analysis of the $>51$ breakdowns observed in these experiments is beyond the scope of this manuscript. Here we summarize only the main characteristics observed, and a fuller account may be found in Masino (2001). Breakdowns occurred when driving both oscillator interneurons (G3 or G4) and coordinating interneurons (G2) (Fig. 6). When driving oscillator interneurons (either G3 or G4 interneurons), two types of breakdowns were observed: those in which the driven half-center oscillator remained functionally intact (Fig. 6A, 25\%) and those in which it was broken apart (Fig. 6B, 75\%). In the former, the paired interneurons of the driven half-center oscillator remained coordinated (intact), whereas coordination between the driven and follower half-center oscillators was lost (Fig. 6A, driven $\mathrm{HN}(\mathrm{L}, 3))$. In the latter breakdowns, the driven oscillator interneuron was isolated from the timing network; the driven half-center oscillators no longer functioned normally (broken), and the interneurons of the follower oscillator were not coordinated to the driven cell (Fig. $6 B$, driven $\operatorname{HN}(\mathrm{R}, 4)$ ). When driving coordinating interneurons (G2 interneurons), all breakdowns observed were similar. The driven G2 cell lost control of the ipsilateral oscillator cells in G3 and G4 and was thus isolated from the timing network (Fig. 6C). The G3 and G4 oscillator interneurons, however, remained coordinated.

\section{DISCUSSION}

In this paper, we have further tested a model of the leech heartbeat timing network (Hill et al., 2002), here referred to as the simple symmetrical model (Fig. $1 B$ ). The model predicts that intersegmental phase differences will be proportional to the period differences between segmental oscillators, and the period of the coupled network will be the period of the faster oscillator. These predictions of the model were borne out in an extensive experimental analysis involving reversible uncoupling and recoupling of the two segmental oscillators (sucrose knife) and split bath applications of agents (myomodulin or $\mathrm{Cs}^{+}$) that modify the period of the segmental oscillators (Masino and Calabrese, 2002b). Thus, under closed-loop conditions of mutual entrainment of the segmental oscillators, the system acts like a symmetrically coupled pair of oscillators that can influence (speed) one

The G3 to G4 phase relationships among these preparations range between approximately $-20 \%$ (G3 driven approximately $-15 \%$ faster than normal) to approximately $+20 \%$ (G3 driven approximately $+15 \%$ slower than normal). $B, \mathrm{G} 4$ oscillator interneurons driven to various cycle periods entrain the timing network over a narrow range of periods faster (approximately $-5 \%$ ) and slower (approximately $+10 \%$ ) than the normal cycle period. There is a near linear relationship (slope) between the G3 to G4 phase relationships and the changes in cycle period. The G3 to G4 phase relationships among these preparations range between approximately $-20 \%$ (G4 driven approximately $-5 \%$ faster than normal) to approximately $+20 \%$ (G4 driven approximately $+10 \%$ slower than normal). $C, \mathrm{G} 2$ coordinating interneurons driven to various cycle periods entrain the timing network over a moderate range of periods both faster (approximately $-15 \%$ ) and slower (approximately $+5 \%$ ) than the normal cycle period. The relationship between the G3 to G4 phase relationships and the changes in cycle period is nearly flat. The G3 to G4 phase relationships among these preparations range between approximately $-15 \%$ ( $\mathrm{G} 2$ driven approximately $-15 \%$ faster than normal) to approximately $+20 \%$ (G3 driven approximately $+5 \%$ slower than normal). 
A Intact Driven Half-Center Oscillator (G3)

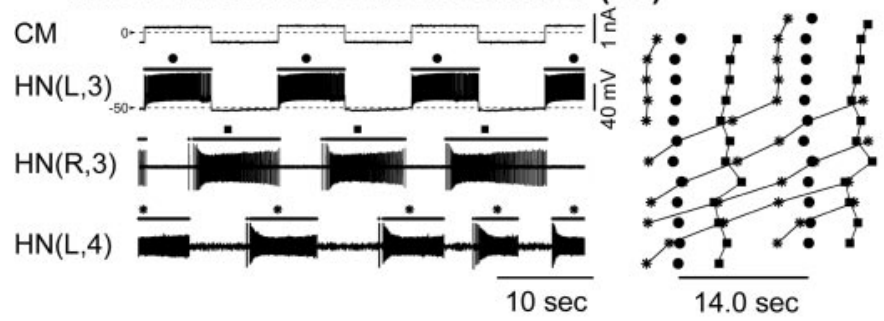

B Isolated Driven HN(R,4) Cell

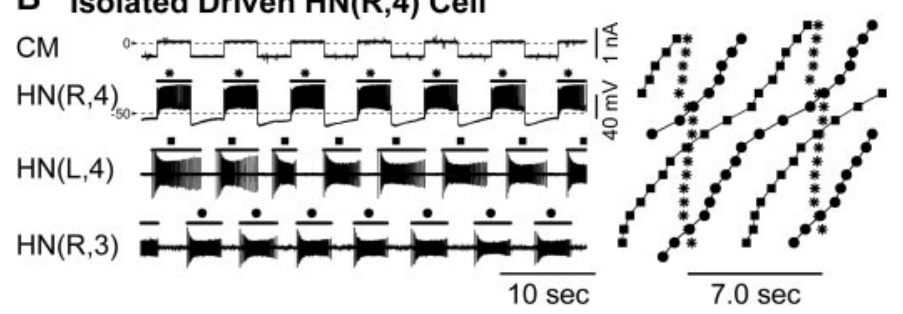

\section{Isolated Driven HN(R,2) Cell}

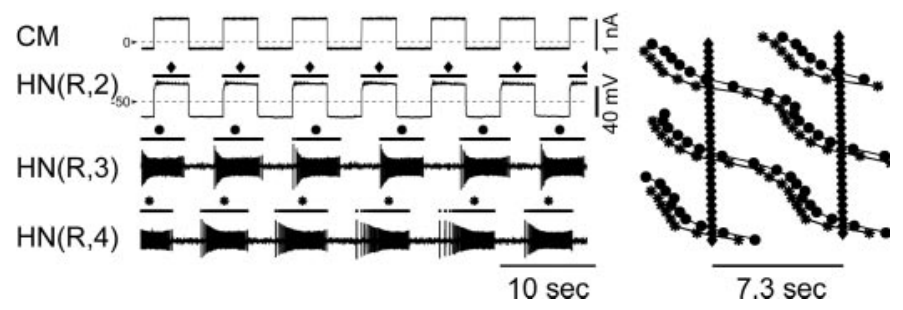

Figure 6. Breakdowns of entrainment when driving oscillator or coordinating interneurons. In this figure, the reference cycle of the actograms are set to the driven period so that the symbols for the driven cell form a vertical column. $A$, The $\mathrm{HN}(\mathrm{L}, 3)$ interneuron is driven by current pulses to a period $(14.0 \mathrm{sec})$ that is much slower than the normal cycle period $(9.1 \mathrm{sec})$. The contralateral G3 oscillator interneuron $[H N(R, 3)]$ maintains a regular phase relationship to the driven interneuron, but the ipsilateral G4 oscillator interneuron $[H N(L, 4)]$ breaks from entrainment and expresses an independent faster period as seen by the symbols (asterisks) in the actogram suddenly drifting dramatically to the left. The G3 half-center oscillator appears to be functioning normally (intact), but coordination between G3 and G4 has broken down at least ipsilateral to the driven interneuron. $B$, The $\mathrm{HN}(\mathrm{R}, 4)$ interneuron is driven by current pulses to a period $(7.0 \mathrm{sec})$ that is much slower than the normal cycle period $(6.2$ $\mathrm{sec})$. The contralateral $\mathrm{G} 4$ oscillator interneuron $[H N(L, 4)]$ does not maintain a regular phase relationship to the driven cell. Because it expresses a faster period, it slowly drifts by the driven G4 oscillator interneuron as seen by the pattern of the symbols (squares) drifting to the left in the actogram. Like the contralateral G4 oscillator interneuron, the ipsilateral $\mathrm{G} 3$ oscillator interneuron $[H N(R, 3)]$ drifts by the driven cell but remains phase locked with the contralateral G4 oscillator interneuron. The G3 to G4 phase relationship is disturbed periodically because of a perturbing influence of the driven G4 oscillator interneuron on its contralateral homolog, which is most easily seen by following the "parallel" paths of the symbols [squares $(\mathrm{HN}(\mathrm{L}, 4)]$ and circles $(H N(R, 3)]$ starting at the top right of the actogram. The G4 half-center oscillator appears to be no longer functioning normally (broken). Coordination between contralateral G4 $[H N(L, 4)]$ and $\mathrm{G} 3[H N(R, 3)]$ oscillator interneurons remains but does not include the driven G4 oscillator interneuron, which thus appears isolated from the rest of the timing network. $C$, The $\operatorname{HN}(\mathrm{R}, 2)$ interneuron is driven by current pulses to a period $(7.3 \mathrm{sec})$ that is faster than the normal cycle period $(7.9 \mathrm{sec})$. The ipsilateral G4 $[H N(R, 4)]$ and $\mathrm{G} 3[H N(R, 3)]$ oscillator interneurons break from entrainment and express an independent slower period as seen by the symbols (asterisks and circles) in the actogram drifting to the right. The ipsilateral oscillator interneurons maintain a regular phase relationship with the G4 oscillator interneuron leading. This relationship between oscillator interneurons is most easily seen by following the parallel sinuous paths another by the removal of inhibition from the coordinating interneurons.

The model, however, makes two simplifying assumptions not borne out by the data. First, it assumes that the coordinating interneurons fire at a constant rate for the entire period that they are not inhibited by the oscillator interneurons. The biological coordinating interneurons show considerable spike frequency adaptation during their burst and do not always fill the interval available for them to fire (Masino and Calabrese, 2002a). Second, it assumes that all spikes in the coordinating interneurons arise at a single initiation site in G4 that is inhibited equally by the ipsilateral G3 and G4 oscillator interneurons. The biological coordinating interneurons initiate at least $15 \%$ of their spikes (mostly during the interval of firing overlap with the G4 oscillator interneuron; see Results) at a G3 initiation site that is inhibited solely by the ipsilateral G3 oscillator interneuron (Masino and Calabrese, 2002a). Moreover, shifts of spike initiation from the G4 site to the G3 site are regularly observed when the activity of the G4 oscillator interneuron is perturbed by injected current.

The simple symmetrical model, furthermore, makes two predictions when tested under open-loop conditions by periodic driving of single oscillator interneurons (Hill et al., 2002). First, the system will respond symmetrically to driving a G3 versus a G4 oscillator interneuron, and second, the system can be entrained during driving only when the driven period is fast enough to allow the driven cell to lead in phase. The deviations of the biological system from the assumptions of the simple symmetrical model motivated the tests of these predictions of the model under the open-loop conditions described here.

In these experiments, all members of the heartbeat timing network, which include both oscillator interneurons and coordinating interneurons, were able to entrain the entire timing network under open-loop (driving) conditions. Nevertheless, the two predictions of the simple symmetrical model were not borne out.

The biological system can be driven to periods in which the driven oscillator clearly lags the follower oscillator by a large margin (Figs. 2, 3, 5A,B), whereas the simple symmetrical model cannot (Hill et al., 2002, their Fig. 8). Spike frequency adaptation during the bursts of the coordinating interneuron, which was not included in the simple symmetrical model, may explain this ability in the biological system. A driven half-center oscillator may be able to slow the follower half-center oscillator, and thus the entire timing network by shifting the high spike frequency portion of the ipsilateral coordinating burst of the interneuron to a point late in the inhibited phase of the follower oscillator interneuron. The timing of inhibition from the coordinating interneuron affects the length of the interburst interval and thus cycle period (Hill et al., 2002) (Fig. 6). High frequency activity of the coordinating interneuron late in the inhibited phase of the oscillator interneuron delays the onset of activity in the oscillator interneuron and thus increases the oscillators cycle period. When this high-frequency portion falls early in the inhibited phase of an oscillator interneuron, however, it has minimal effects, as would occur in both G3 and G4 oscillator interneurons when there is no phase difference between the oscillators.

of the symbols [circles $(H N(R, 3)$ and asterisks $(H N(R, 4)]$ starting at the top left of the actogram. The kinks in this path appear to be caused by repeated weak (relative) entrainment by the driven G2 (diamonds) oscillator interneuron. The driven G2 coordinating interneuron no longer entrains the timing network, but coordination between G3 and G4 remains at least ipsilateral to the driven interneuron. 
The driving experiments presented here also contradict the assumption that the timing network functions exclusively in the symmetric mode because the G3 and G4 oscillator interneurons differ in their ability to entrain the network and in the consequences for the phase relationships within the network. The driven G3 oscillator entrains the timing network over a broader range of cycle periods than does the driven G4 oscillator (Fig. 5, compare $A, B)$. The $\mathrm{G} 4$ oscillator is particularly restricted in entraining the network to periods shorter than the undriven period. The most parsimonious explanation for this functional asymmetry is the known asymmetry in the circuitry of the timing network (Fig. 1A). The G3 oscillator interneurons should have better control over the entire timing network and thus be able to entrain the network over a broader range of cycle periods than the G4 oscillator interneurons, because the G3 oscillator interneurons inhibit the primary (G4) and secondary (G3) spike initiation sites of the coordinating interneurons. The G4 oscillator interneurons should be less able to control the entire timing network and thus drive the network over a narrower range of cycle periods than the G3 oscillator interneurons, because the G4 oscillator interneurons only inhibit the primary spike initiation site of the coordinating interneurons in G4.

Despite this experimentally demonstrable asymmetry in the range of entrainment of the two oscillators, each driven oscillator produces similar ranges of phase relationships across different ranges of driven periods (Fig. 5, compare $A, B$ ). One way of looking at this similarity is to hypothesize that the phase difference between the two oscillators is more sensitive to a change in driving period when a G4 oscillator is driven, compared with a G3 oscillator. Viewing the similarity from an opposing point-ofview may be more heuristic; the G4 oscillator requires a bigger phase difference to entrain the G3 oscillator to its period. As an example, consider our previous modeling results using the simple symmetric model (Hill et al., 2002). This model indicates that one oscillator influences the other by removing inhibition from the other. When the G3 oscillator leads in phase, its net effect is to truncate inhibition from the coordinating interneurons to the G4 oscillator, thus speeding it. The bigger the phase lead by the G3 oscillator, the more inhibition is removed, and thus the more speeding of the G4 oscillator is effected. In the symmetric model, the G4 oscillator would act equivalently when it leads. Consider now that the G4 oscillator controls only the G4 initiation sites of the coordinating interneurons. Its net effect when it leads in phase is not to truncate the inhibition to the $\mathrm{G} 3$ oscillator but to reduce it (initiation shifts to the slower G3 site in the coordinating interneurons (Masino and Calabrese, 2002a, their Fig. 4). Thus, to effect a similar reduction in inhibition to the other oscillator, a G4 oscillator must assume a bigger phase lead than a G3 oscillator. To substantiate this line of thinking, we are pursuing a model of the heartbeat timing network in which the coordinating interneurons have two asymmetric spike initiation sites, each showing spike frequency adaptation, and the actual asymmetric network connectivity is implemented.

We also observed entrainment when driving individual coordinating interneurons. The range of periods over which the coordinating interneurons were able to entrain the oscillators, although smaller than that of the G3 oscillator interneurons, was still substantial, but they had little effect of the phase relationship between the oscillators. These observations, taken together, indicate that coupling between the oscillators in the heartbeat timing network is strong, but that phase relations are determined by properties (membrane or synaptic) inherent to the oscillator interneurons.

\section{How strong is the intersegmental coupling between half-center oscillators in G3 and G4?}

The most surprising result observed during breakdowns in entrainment when driving oscillator interneurons is that the G3 and/or G4 oscillator often broke down with the driven cell in some cases isolated from the otherwise mutually entrained network (most breakdowns driving a G4 oscillator interneuron) or side to side coordination is disrupted whereas intersegmental coordination is not (most breakdowns driving a G3 oscillator interneuron). These results suggest that the intersegmental synaptic connections that link the G3 and G4 oscillators via the coordinating interneurons are of comparable functional weight as those that link the oscillator interneurons into half-center oscillators. Although it is tempting to make simple assertions about the mechanisms by which a dynamic neural circuit functions, the complexity of network dynamics often makes this difficult. The leech heartbeat timing network, although quite simple in terms of its connectivity pattern (Fig. $1 A$ ), defies simple explanation. The pattern of synaptic connections within the network permit it to function potentially in two modes: symmetric or asymmetric. The network, however, behaves both symmetrically and asymmetrically depending on the conditions. Under closedloop conditions of mutual entrainment, the timing network behaves "symmetrically". There are indications of asymmetry, however, even under this condition (i.e., the phase of the coordinating interneurons is more tightly regulated by the G3 oscillator than by the G4 oscillator, and the activity in the coordinating interneurons overlaps more with activity in the G3 oscillator interneurons than with G4 oscillator interneurons) (Masino and Calabrese, 2002a). Conversely, as shown here the timing network behaves "asymmetrically" when it is tested under open-loop conditions. The asymmetry is not complete; the G4 oscillator interneurons are able to entrain the network, but over a much more limited period range than the G3 oscillator. The heartbeat timing network thus does not function solely in one mode or the other, but rather as a hybrid that seems to shift dynamically between the two modes, depending on the current conditions of the network. Moreover, the breakdowns observed when driving oscillator interneurons indicate that the strength of intersegmental synaptic coupling is comparable with the synaptic coupling with the segmental half-center oscillators. Thus, the heartbeat timing network may best be viewed as a dynamic whole rather than as a system of two coupled, but otherwise independent oscillators.

\section{REFERENCES}

Angstadt JD, Calabrese RL (1989) A hyperpolarization-activated inward current in heart interneurons of the medicinal leech. J Neurosci 9:2846-2857.

Braun G, Mulloney B (1995) Coordination in the crayfish swimmeret system: differential excitation causes changes in intersegmental phase. J Neurophysiol 73:880-885.

Calabrese RL, Nadim F, Olsen $\varnothing \mathrm{H}$ (1995) Heartbeat control in the medicinal leech: a model system for understanding the origin, coordination, and modulation of rhythmic motor patterns. J Neurobiol 27:390-402.

Cohen AH (1987) Effects of oscillator frequency on phase-locking in the lamprey central pattern generator. J Neurosci Methods 21:113-125.

Grillner S, Matsushima T, Wadden T, Tegner J, El Manira A, Wallen P (1993) The neurophysiological bases of undulatory locomotion in vertebrates. Semin Neurosci 5:17-27.

Hill AAV Lu, Masino MA, Calabrese RL (2001) A model of a segmental oscillator in the leech heartbeat neuronal network. J Comput Neurosci 10:281-302.

Hill AAV, Masino MA, Calabrese RL (2002) Model of intersegmental 
coordination in the leech heartbeat neuronal network. J Neurophysiol 87:1586-1602.

Kotaleski JH, Lansner A, Grillner S (1999a) Neural mechanisms potentially contributing to the intersegmental phase lag in lamprey. II. Hemisegmental oscillations produced by mutually coupled excitatory neurons. Biol Cybern 81:299-315.

Kotaleski JH, Grillner S, Lansner A (1999b) Neural mechanisms potentially contributing to the intersegmental phase lag in lamprey. I. Segmental oscillations dependent on reciprocal inhibition. Biol Cybern 81:317-330.

Marder E, Calabrese RL (1996) Principles of rhythmic motor pattern generation. Physiol Rev 76:687-717.

Masino MA (2001) Intersegmental coordination in the leech heartbeat central pattern generator. PhD dissertation, Emory University.

Masino MA, Calabrese RL (2002a) Phase relations between segmentally organized oscillators in the leech heartbeat pattern generating network. J Neurophysiol 87:1572-1585.

Masino MA, Calabrese RL (2002b) Inherent period differences between segmental oscillators produce intersegmental phase differences in the leech heartbeat pattern generating network. J Neurophysiol 87: $1603-1615$.

Mulloney B (1997) A test of the excitability-gradient hypothesis in the swimmeret system of crayfish. J Neurosci 17:1860-1868.

Nadim F, Calabrese RL (1997) A slow outward current activated by FMRFamide in heart interneurons of the medicinal leech. J Neurosci 17:4461-4472.

Nadim F, Olsen $\varnothing \mathrm{H}$, Calabrese RL (1995) Modeling the leech heartbeat elemental oscillator I. Interactions of intrinsic and synaptic currents J Comput Neurosci 2:215-235.

Peterson EL (1983a) Generation and coordination of heartbeat timing oscillation in the medicinal leech. I Oscillation in isolated ganglia J Neurophysiol 49:611-626.

Peterson EL (1983b) Generation and coordination of heartbeat timing oscillation in the medicinal leech. II Intersegmental coordination J Neurophysiol 49:627-638.

Peterson EL, Calabrese RL (1982) Dynamic analysis of a rhythmic neural circuit in the leech Hirudo medicinalis. J Neurophysiol 47:256-271.

Pittendrigh CS (1974) Circadian oscillations in cells and the circadian organization of multicellular systems. In: The neurosciences third study program (Schmitt FO, Worden FG, eds), pp 437-458. Cambridge: MIT.

Sigvardt KA (1993) Intersegmental coordination in the lamprey central pattern generator for locomotion. Semin Neurosci 5:3-15.

Skinner FK, Mulloney B (1998) Intersegmental coordination of limb movements during locomotion: mathematical models predict circuits that drive swimmeret beating. J Neurosci 18:3831-3842.

Stein PS (1971) Intersegmental coordination of swimmeret motoneuron activity in crayfish. J Neurophysiol 34:310-318.

Wadden T, Hellgren J, Lansner A, Grillner S (1997) Intersegmental coordination in the lamprey: simulations using a network model without segmental boundaries. Biol Cybern 76:1-9.

Wallén P, Ekeberg O, Lansner A, Brodin L, Traven H, Grillner S (1992) A computer-based model for realistic simulations of neural networks. II. The segmental network generating locomotor rhythmicity in the lamprey J Neurophysiol 68:1939-1950. 